\title{
Electron Stability and Negative-Tetron Luminescence in Free-Standing Colloidal n-Type CdSe/CdS Quantum Dots
}

Kimberly H. Hartstein, Christian S. Erickson, Emily Y. Tsui, ${ }^{\S}$ Arianna Marchioro, and Daniel R. Gamelin*

Department of Chemistry, University of Washington, Seattle, Washington 98195-1700, United States

\begin{abstract}
We examine the effects of CdS shell growth on photochemical reduction of colloidal CdSe quantum dots (QDs) and describe the spectroscopic properties of the resulting n-type CdSe/CdS QDs. CdS shell growth greatly slows electron trapping. Because of this improvement complete two-electron occupancy of the 1Se conduction-band orbital is achieved in $\mathrm{CdSe} / \mathrm{CdS}$ QDs and found to be much more stable than in past experiments. Simultaneous photoluminescence at two different energies is now observed from QDs possessing two excess conduction-band electrons, reflecting competing recombination of discretized $1 \mathrm{~S}_{\mathrm{e}}$ and $1 \mathrm{P}_{\mathrm{e}}$ conduction-band electrons within photogenerated four-carrier negative tetrons (three electrons and one hole). Stable occupancy of the $1 \mathrm{P}_{\mathrm{e}}$ level is not achievable under these conditions, and possible reasons are discussed. The stability and accessibility of these multielectron configurations, and the facile spectroscopic detection of negative tetrons, both make photodoped core/shell QDs attractive for exploring the physical properties of free-standing heavily n-doped colloidal CdSe-based QDs.
\end{abstract}

\section{Introduction}

A fundamental understanding of the electronic structures of colloidal semiconductor quantum dots (QDs) is vital to the application of this class of materials in future technologies. Attractive due to their size-tunable physical properties and solution processability, free-standing colloidal QDs suffer from surface defects that have presented longstanding barriers to many practical applications by limiting luminescence quantum yields, affecting Fermi levels, and ultimately impacting performance in various QD-based devices. Surface modification is commonly employed to mitigate the influence of surface traps, most often in the form of passivating smallmolecule surface ligation or shell overgrowth. Through judicious selection of the core and shell parameters, the latter can also be used to strategically tune the material's electronic structure for enhancement of target functional properties. For example, the $\mathrm{CdSe} / \mathrm{CdS}$ core/shell 
heterostructure has long attracted interest because of its quasi-type II band alignment, in which conduction-band electrons $\left(e_{\mathrm{CB}}^{-}\right)$delocalize throughout the heterostructure but valence-band holes $\left(h_{\mathrm{VB}}^{+}\right)$localize at the CdSe core. ${ }^{1-11}$ This spatial separation of charge carriers prolongs exciton radiative lifetimes, slows Auger recombination, enhances photocatalysis, suppresses luminescence intermittency ("blinking"), and eliminates some nonradiative surface recombination pathways.

Electronic doping is a powerful approach for studying the electronic structures of semiconductor nanocrystals. ${ }^{12}$ Among the various methods for introducing excess delocalized charge carriers, photodoping has proven particularly useful for achieving reversible and quantifiable tuning of electron occupancies in free-standing oxide and chalcogenide QDs. ${ }^{12-22}$ Here, we use photodoping to incorporate excess $e_{\mathrm{CB}}^{-}$into colloidal $\mathrm{CdSe} / \mathrm{CdS}$ core/shell QDs with various shell thicknesses. We show that the excess conduction-band electrons become increasingly stable at room temperature with increasing CdS shell thickness. This stability allows detailed chemical and spectroscopic characterization of the resulting n-doped core/shell QDs in the absence of electrodes, excess reductant, or other potential perturbations. In QDs containing two extra $e_{\mathrm{CB}}^{-}$, we observe separate photoluminescence (PL) bands from recombination of discretized $1 \mathrm{~S}_{\mathrm{e}}$ and $1 \mathrm{P}_{\mathrm{e}}$ electrons as well as corresponding dynamic signatures of four-carrier negative tetrons (three $e_{\mathrm{CB}}^{-}$and one $h_{\mathrm{VB}}^{+}$). Discrete $1 \mathrm{~S}_{\mathrm{e}}$ and $1 \mathrm{P}_{\mathrm{e}} \mathrm{PL}$ bands have been observed in other CdSe-based QDs under electrochemical bias, ${ }^{23,24}$ and the dynamics of negative tetrons have been described for CdSe/CdS QDs. ${ }^{22,25}$ Here, we quantify these two phenomena together in conjunction with chemical titration analysis of the extent of n-doping to demonstrate the relationship between them. The findings presented here improve our understanding of electronic materials relevant to various QD optoelectronic technologies and point to the use of photodoped n-type $\mathrm{CdSe} / \mathrm{CdS}$ core/shell QDs for future fundamental electronic-structure studies of multielectron QDs.

\section{Results and analysis}

Photodoping, Electron Stability, and Electron Titration. Figure 1 shows TEM images of a representative series of $\mathrm{CdSe} / \mathrm{xCdS}$ core/shell QDs prepared from the same CdSe cores but with different CdS shell thicknesses of $x=2,4$, and 7 monolayers. The average QD diameter increases with increasing shell thickness, as does the nanocrystal faceting, and a small increase in the size distribution is also observed. 


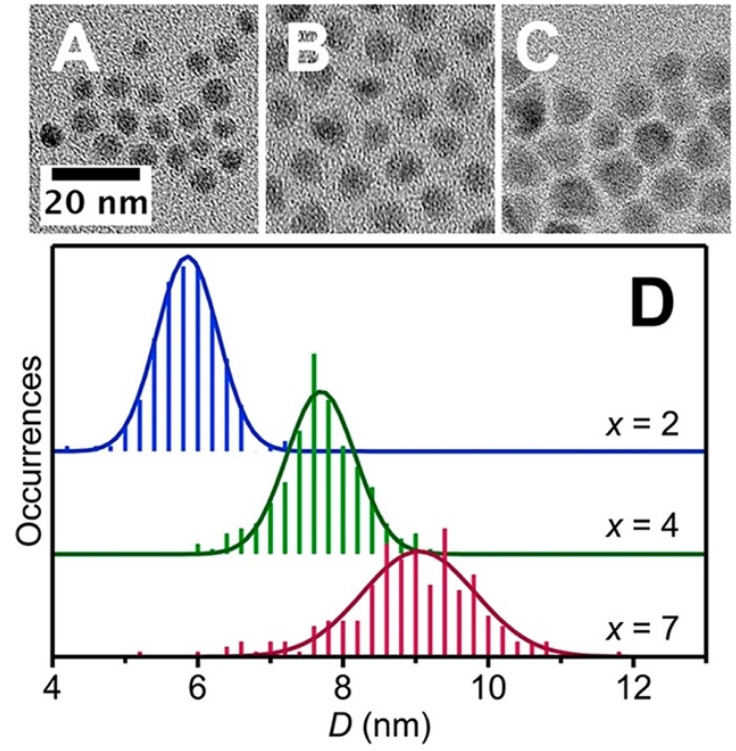

Figure 1. (A-C) TEM images of representative CdSe/xCdS QDs with $\mathrm{x}=2$, 4, and 7, respectively, grown on $\mathrm{d}=$ $4.3 \mathrm{~nm}$ CdSe cores. (D) Size histograms for each core/shell sample, based on analysis of more than 200 QDs each. The reported numbers of CdS shell monolayers (x) are calculated from the average TEM diameters by assuming each monolayer has a thickness of $0.35 \mathrm{~nm}^{26}$

Core/shell QDs were reduced photochemically following the approach described in detail previously, ${ }^{14,19}$ in which $\mathrm{Li}$-[Et $\left.{ }_{3} \mathrm{BH}\right]$ pre-reduces the QD surfaces, followed by photoexcitation and hole capture. Figure 2 plots normalized absorption and continuous-wave photoluminescence (CW PL) spectra of as-prepared (solid) and maximally photodoped (dashed) CdSe/xCdS QDs with increasing shell thickness (top to bottom). The combination of large valence-band offset and small conduction-band offset between CdSe and CdS leaves holes largely confined to the CdSe volumes but permits delocalization of electrons throughout the entire heterostructure volumes, ${ }^{27}$ resulting in a redshift in absorption and PL energies of the as-prepared QDs with increasing CdS shell thickness. The PL quantum yield (QY) of the asprepared QDs also increases, from $\sim 3 \%$ at $\mathrm{x}=0$ to $\sim 73 \%$ at $\mathrm{x}=7$. Resonant photoexcitation of the CdSe feature still leads to photodoping (see Figure S1), demonstrating hole capture at the surface despite confinement to the CdSe core. 

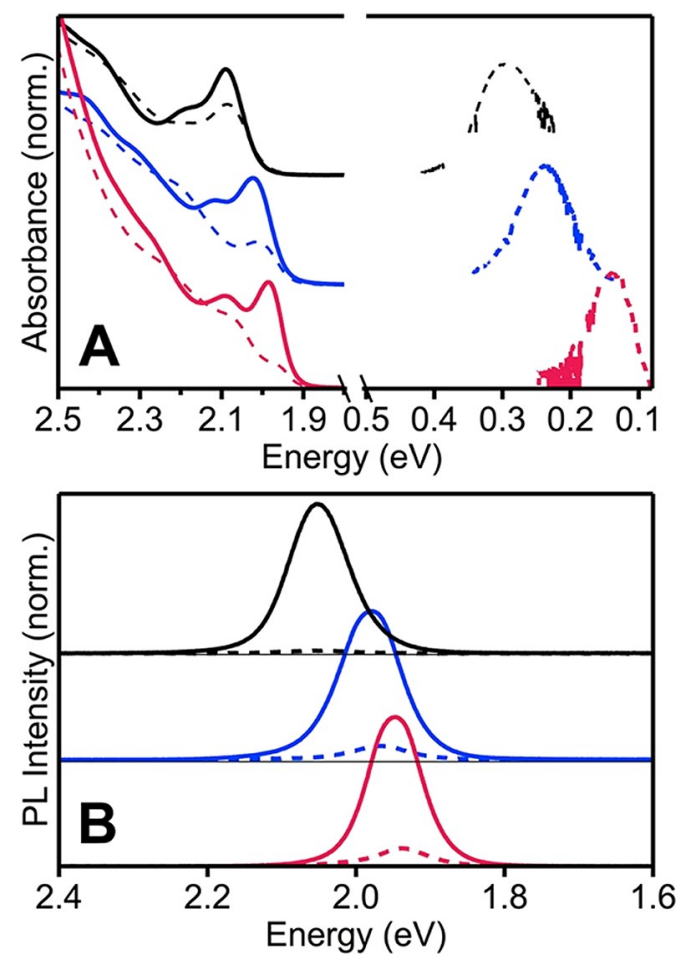

Figure 2. (A) Visible and infrared $\left(1 \mathrm{~S}_{\mathrm{e}}-1 \mathrm{P}_{\mathrm{e}}\right)$ electronic absorption and $(\mathrm{B})$ visible CW PL spectra of as-prepared (solid) and maximally photodoped (dashed) $\mathrm{CdSe} / \mathrm{xCdS}$ QDs with $\mathrm{x}=0,2$, and 7 monolayers of CdS shell (from top to bottom) deposited on $\mathrm{d}=4.3 \mathrm{~nm}$ CdSe QD cores. Major solvent peaks have been removed from the IR spectra for clarity. With increasing shell thickness, $\left\langle n_{\max }\right\rangle$ increases. CW PL intensity is still observed at $\left\langle\mathrm{n}_{\max }\right\rangle$ for the shelled samples.

Concomitant with these changes, the maximum extent of photodoping that can be achieved also increases with increasing shell thickness, as gauged by the bleach of the first excitonic absorption peak $\left(1 \mathrm{~S}_{\mathrm{h}}-1 \mathrm{~S}_{\mathrm{e}}\right)$. In the core CdSe QDs $(\mathrm{x}=0)$, this absorbance is reduced by only $\sim 35 \%$ before further photoexcitation no longer generates additional $1 \mathrm{~S}_{\mathrm{h}}-1 \mathrm{~S}_{\mathrm{e}}$ bleach, whereas in the largest CdSe/CdS QDs ( $\mathrm{x}=7$ ), the first exciton is bleached by $\sim 90 \%$ at maximum photodoping. The average number of excess $e_{\mathrm{CB}}^{-}$per QD $(\langle n\rangle)$ can be quantified by analyzing the extent of this bleach using the relationship $\langle\mathrm{n}\rangle=|(2 \Delta \mathrm{A} / \mathrm{A} 0)|{ }^{14,17,19,21}$ At maximum photodoping, we define $\langle n\rangle=\left\langle n_{\max }\right\rangle$. The CdSe QD cores without any shell reach only $\left\langle n_{\max }\right\rangle$ $\sim 0.7$, but depositing just two monolayers of CdS shell increases $\left\langle n_{\max }\right\rangle$ to 1.6 , and at 7 shell monolayers $\left\langle n_{\max }\right\rangle=1.8$. We have previously shown that $\left\langle n_{\max }\right\rangle$ in CdSe QDs reflects the steady-state balance between photoreduction and electron trapping and that increases in $\left\langle n_{\max }\right\rangle$ correlate with stabilization of the conduction-band-edge potential to reduce the number of midgap electron traps. ${ }^{21,28}$ We have also shown that even though midgap electron traps below the Fermi level are filled by photodoping, new midgap traps appear spontaneously on long time scales because of trap-state fluctuations. ${ }^{21}$ Following these observations, the trend of increasing $\left\langle n_{\max }\right\rangle$ with CdS shell growth is attributed to (1) reduction of the number of midgap surface 
electron traps through physical passivation, and (2) shifting of the conduction-band edge to more positive redox potentials relative to the electron-trap-state distribution through relaxation of electron quantum confinement. ${ }^{21}$ With these two effects, conduction-band electrons have access to fewer midgap surface traps after CdS shell growth, leading to lower electron-trapping rates (vide infra) and hence greater values of $\left\langle n_{\max }\right\rangle$.

In the unshelled $(\mathrm{x}=0) \mathrm{CdSe}$ QDs, the CW PL is almost completely quenched at $\left\langle n_{\max }\right\rangle$ due to efficient Auger recombination. Statistically, each thin-shelled $(x=2) \mathrm{CdSe} / \mathrm{CdS}$ QD contains at least one $e_{\mathrm{CB}}^{-}$at $\left\langle n_{\max }\right\rangle \sim 1.6$, and yet considerable PL intensity remains. The thickshelled $(\mathrm{x}=7)$ QDs also still luminesce even at $\left\langle n_{\max }\right\rangle \sim 1.8$. The remaining CW PL intensity is attributed to the reduced nonradiative Auger decay rate of negative trions in these QDs, due to the combination of relaxed quantum confinement ${ }^{15}$ and increased spatial separation between charge carriers ${ }^{8}$ with increasing CdS shell thickness.

The fluctuations described above cause new midgap electron traps to appear spontaneously over very long time scales, causing slow electron trapping even under rigorously anaerobic conditions. ${ }^{19,21}$ This slow trapping is manifested as an extremely slow recovery of excitonic absorption after photodoping. ${ }^{21}$ Detailed studies have demonstrated that this trapping follows unimolecular reaction kinetics and hence cannot be attributed to the trivial scenario of diffusive oxidants in solution. ${ }^{21}$ Figure 3 summarizes the slow anaerobic absorption recovery observed for photodoped CdSe/xCdS QDs $(\mathrm{x}=2,4,7)$. The data are plotted as the normalized first-excitonic absorption bleach $(-\Delta \mathrm{A} / \mathrm{A} 0)$ vs time. Fitting these data yields the electron decay time constants $\left(\tau_{\mathrm{D}}\right)$ plotted in the inset of Figure 3. These data show that increasing the CdS shell thickness reduces the rate of electron trapping markedly. For example, $\tau_{\mathrm{D}} \sim 15 \mathrm{~min}$ when $\mathrm{x}=2$, but increases to several hours for $\mathrm{x}=7$. Photodoped CdSe/7CdS QDs are thus remarkably stable as free-standing colloidal n-type nanocrystals.

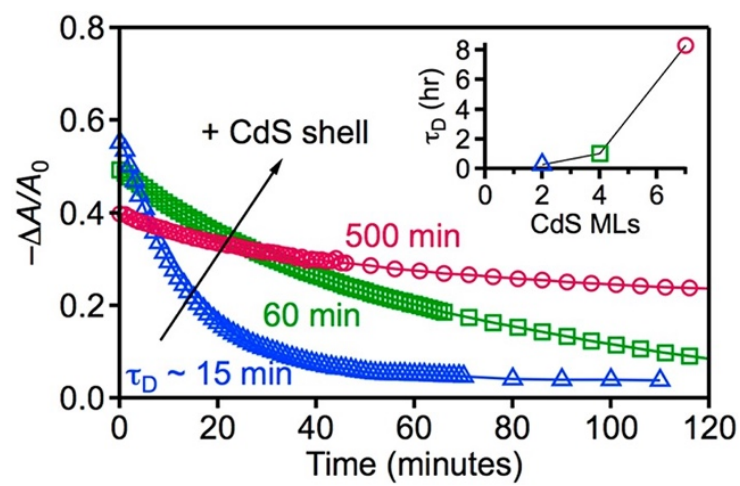

Figure 3. Anaerobic $e_{\mathrm{CB}}^{-}$decay kinetics for $\mathrm{CdSe} / \mathrm{xCdS}$ QDs with $\mathrm{x}=2$ (blue open triangle), 4 (green open square), and 7 (red open circle) monolayers, measured in solution at room temperature. The data points represent the normalized absorption at the maximum of the first excitonic absorption transition $\left(1 \mathrm{~S}_{\mathrm{h}}-1 \mathrm{~S}_{\mathrm{e}}\right)$ for each sample. The solid lines show exponential fits to the data, which yield the electron decay time constants $\left(\tau_{\mathrm{D}}\right)$ shown. 
Because the history of the QDs can affect the electron trapping rates, ${ }^{21}$ these samples were prepared and handled under identical conditions for these measurements (see Experimental Methods). The small differences in $\langle\mathrm{n}\rangle$ at $\mathrm{t}$ $=0$ reflect sample handling and do not impact the primary conclusions drawn from these data. Inset: Plot of $\tau_{\mathrm{D}}$ vs CdS shell thickness.

The high stability of these excess conduction-band electrons enables the use of chemical titration as an independent measure of $\langle n\rangle .{ }^{14}$ Previous attempts to titrate large $(\mathrm{d}=$ $7.0 \mathrm{~nm}$ ), maximally photodoped CdSe QDs were complicated by the presence of additional trapped electrons, presumably at surface sites. ${ }^{14}$ Figure 4 shows electronic absorption spectra of maximally photodoped CdSe/7CdS QDs collected during titration with the outer-sphere chemical oxidant, $\left[\mathrm{FeCp}_{2}{ }_{2}\right]-\left[\mathrm{BArF}_{4}\right]$. With each aliquot of oxidant, the absorption recovers partially. The inset plots $-\Delta \mathrm{A} / \mathrm{A} 0$ vs added equivalents of $\left[\mathrm{FeCp}_{2}\right]^{+}$and reveals a linear dependence of the absorption recovery on added oxidant. The intercept of these data with the $\mathrm{x}$ axis indicates $\langle n\rangle \sim 2$. This result is consistent with the spectroscopic estimate of $\langle n\rangle=1.8$ for the same sample. The linearity of these titration data shows that all titratable electrons contribute equally to the absorption bleach, within experimental uncertainty. These data show that few if any electrons reside on the surfaces of these CdSe/7CdS QDs, consistent with nearly complete elimination of midgap electron traps as discussed above.

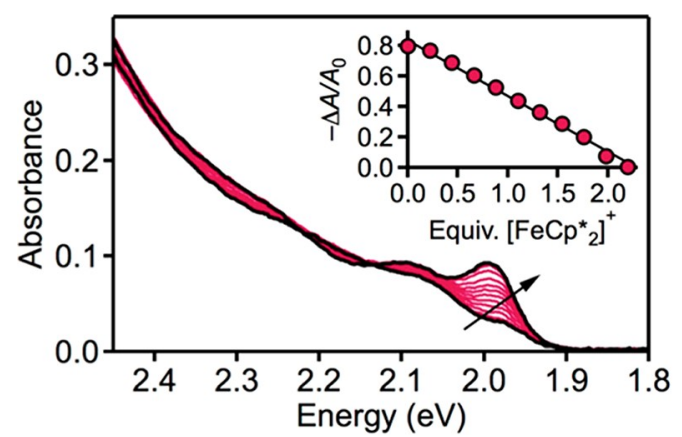

Figure 4. Absorption spectra of maximally photodoped $\mathrm{CdSe} / 7 \mathrm{CdS}$ QDs collected during titration with $\left[\mathrm{FeCp}^{* 2}\right]\left[\mathrm{BArF}_{4}\right]$. Inset: Normalized absorption bleach at the first excitonic maximum vs added $\left[\mathrm{FeCp}^{* 2}\right]^{+}$ equivalents. The titration demonstrates that the maximally photodoped QDs contain an average of $\langle\mathrm{n}\rangle \sim 2$.

Negative-Tetron Luminescence. Figure 5 shows a more detailed view of the absorption and PL changes that accompany photodoping of one representative sample of CdSe/2CdS QDs. Spectra are plotted for these QDs photodoped to $\langle n\rangle=0.0,0.4$, and 1.6. In addition to the increasing bleach of the $1 \mathrm{~S}_{\mathrm{h}}-1 \mathrm{~S}_{\mathrm{e}}$ absorption feature with increasing $\langle n\rangle$, the main PL peak (ascribed to radiative $1 \mathrm{~S}_{\mathrm{h}}-1 \mathrm{~S}_{\mathrm{e}}$ recombination) red-shifts with photodoping, for example, by $\sim 5$ $\mathrm{meV}$ at $\langle n\rangle=0.4$, consistent with emission contributions from negative trions. ${ }^{29}$ At a value of $\langle n\rangle=1.6$, this main feature has shifted by $\sim 25 \mathrm{meV}$, and a second higher-energy PL band appears. This additional PL feature has been observed in CdSe and CdSe/CdS QD films held 
under an applied potential and was assigned to emission involving higher-energy $1 \mathrm{P}_{\mathrm{e}}$ electrons. ${ }^{23,24}$
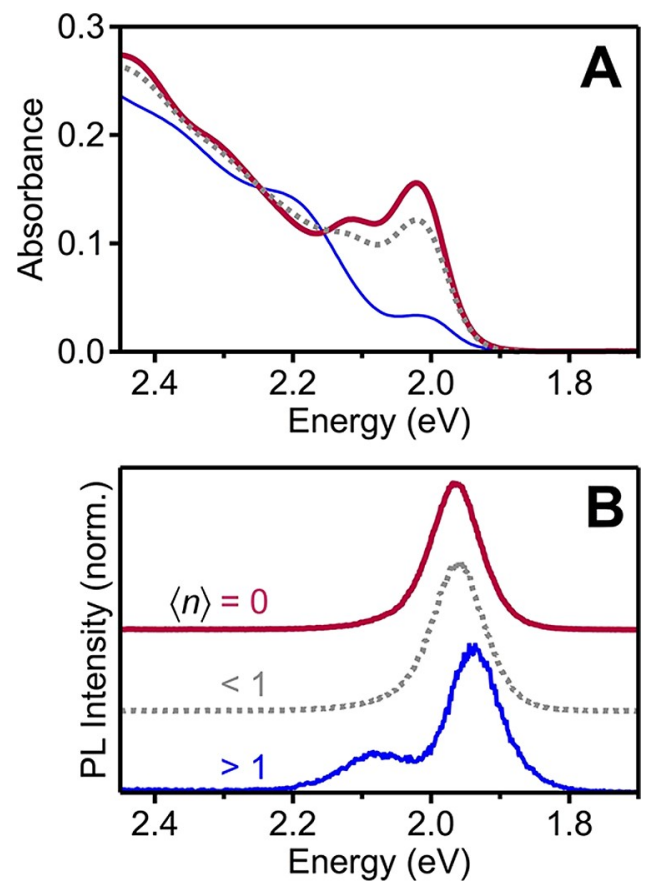

Figure 5. (A) Absorption of CdSe $/ 2 \mathrm{CdS}$ QDs measured at $\langle\mathrm{n}\rangle=0$ (thick solid), $\langle\mathrm{n}\rangle=0.4$ (dashed) and $\left\langle\mathrm{n}_{\max }\right\rangle=$ 1.6 (thin solid). (B) PL spectra of the samples from panel A. At $\langle\mathrm{n}\rangle>1$, a second higher energy PL peak is observed.

Figure 6A plots PL spectra for a series of CdSe/xCdS QDs $(\mathrm{x}=0,2,7)$ collected after photodoping to $\left\langle n_{\max }\right\rangle$ in each case. All three spectra show evidence of an upper PL band. $\left\langle n_{\max }\right\rangle$ is only 0.7 for the $\mathrm{x}=0$ sample, so the $1 \mathrm{P}_{\mathrm{e}}$ orbital is essentially unoccupied at steady state (see Figure S7). Moreover, these unshelled CdSe QDs exhibit negligible CW PL at $\left\langle n_{\max }\right\rangle$ (Figure 2). Nonetheless, their PL spectrum can be obtained by integrating just the first $800 \mathrm{ps}$ of their time-resolved PL, and a small higher-energy PL band is then observed. PL spectra of the CdSe/2CdS QDs were discussed in Figure 5 and show a well-resolved higher-energy band at high photodoping levels. For the CdSe/7CdS QDs, the PL is much stronger, and the upper PL band is again observed. In this sample, the two bands are closer in energy because of relaxed quantum confinement, and they partially overlap.

Figure 6B plots the energy difference between the two PL peaks in Figure 6A $\left(\Delta \mathrm{E}_{\mathrm{P}-\mathrm{S}}(\mathrm{PL})\right)$ vs the energy of the first excitonic absorption maximum $\left(\mathrm{E}_{1 \mathrm{Sh}-1 \mathrm{Se}}\right)$ for these three samples, along with the energy difference between $1 \mathrm{~S}_{\mathrm{e}}$ and $1 \mathrm{P}_{\mathrm{e}}$ one-electron states within the conduction band, as probed by intraband absorption in the infrared ( $\Delta \mathrm{E}_{\mathrm{P}-\mathrm{S}}(\mathrm{IR})$, Figure $\left.2 \mathrm{~A}\right)$. Both energies decrease with decreasing electron confinement upon increasing the CdS shell thickness, but $\Delta \mathrm{E}_{\mathrm{P}-\mathrm{S}}(\mathrm{PL})$ is consistently smaller than $\Delta \mathrm{E}_{\mathrm{P}-\mathrm{S}}(\mathrm{IR})$. The difference between 
$\Delta \mathrm{E}_{\mathrm{P}-\mathrm{S}}(\mathrm{PL})$ and $\Delta \mathrm{E}_{\mathrm{P}-\mathrm{S}}(\mathrm{IR})$ reflects the additional Coulomb interactions present in the initial and final states of the luminescence transitions that are not present in the IR excited states. This correlation thus supports the assignment of this upper PL band to recombination involving $1 \mathrm{P}_{\mathrm{e}}$ electrons in QDs containing multiple excess conduction-band electrons. We note that although the trend in Figure 3 would suggest even greater $e_{\mathrm{CB}}^{-}$stability with increasing CdS shell thickness, the data in Figure 6 show that the upper PL band becomes increasingly obscured by the lower PL band with increasing CdS shell thickness. For this reason, CdSe/xCdS QDs with even thicker CdS shells than $\mathrm{x}=7$ were not explored in this study.
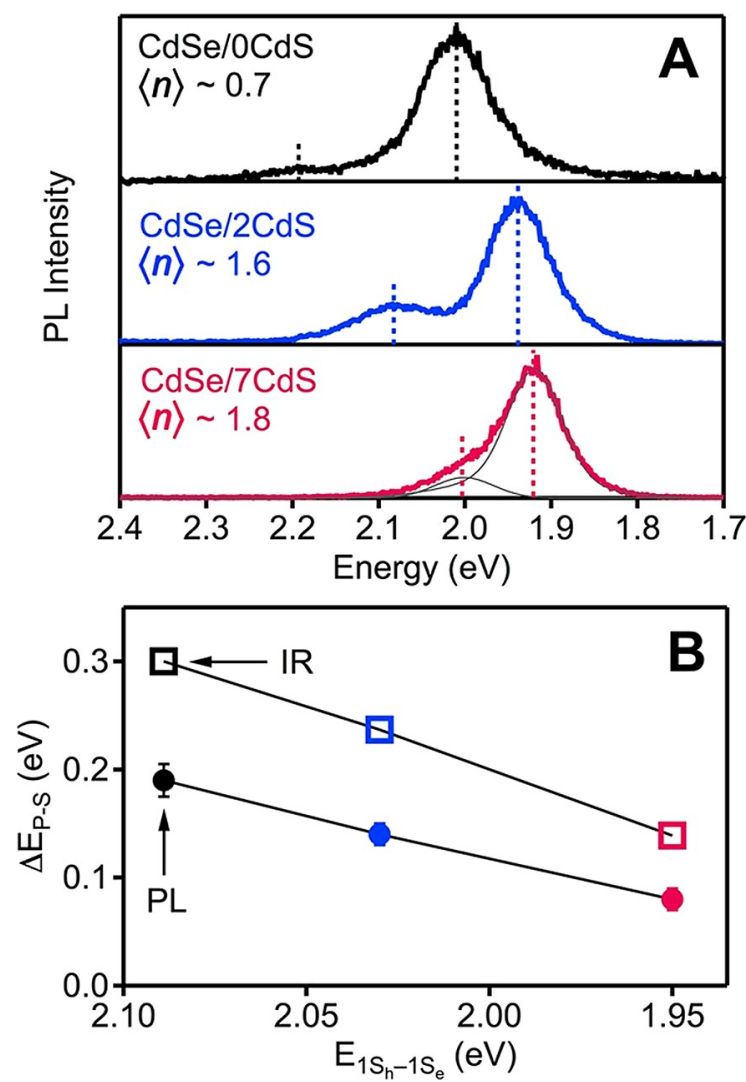

Figure 6. (A) PL spectra for maximally photodoped CdSe/xCdS QDs, where $x=0,2$, and 7 monolayers. Values of $\langle n\rangle$ are indicated on each spectrum. The dotted lines indicate the $1 \mathrm{~S}_{\mathrm{e}}$ and $1 \mathrm{P}_{\mathrm{e}}$ emission energies in each spectrum. (B) $\Delta \mathrm{E}_{\mathrm{P}-\mathrm{S}}$ values determined from IR intraband absorption (Figure 2A) and visible PL (panel A of this figure), plotted vs the energy of the first excitonic absorption feature (E $\left.1_{\mathrm{Sh}-1 \mathrm{Se}}\right) . \Delta \mathrm{E}_{\mathrm{P}-\mathrm{S}}(\mathrm{IR})$ and $\Delta \mathrm{E}_{\mathrm{P}-\mathrm{S}}(\mathrm{PL})$ both decrease with decreasing quantum confinement, and the difference between the two reflects the additional Coulomb interactions probed by the PL measurement. Error bars on $\Delta \mathrm{E}_{\mathrm{P}-\mathrm{S}}(\mathrm{PL})$ values reflect estimated uncertainties from the peak fitting. $\Delta \mathrm{E}_{\mathrm{P}-\mathrm{S}}(\mathrm{IR})$ error bars are smaller than the data points themselves.

Figure 7A shows a streak-camera image describing the PL decay dynamics of the CdSe/2CdS QDs from Figure 5 possessing $\langle n\rangle=\left\langle n_{\max }\right\rangle=1$.6. The integrated $1 \mathrm{~S}_{\mathrm{e}}$ and $1 \mathrm{P}_{\mathrm{e}} \mathrm{PL}$ intensities are plotted vs time in Figure 7B. Although similar, the decay dynamics for the two features do differ slightly. This difference is attributable to the presence of discrete subsets of QDs with $n$ 
$=1$ and $\mathrm{n}=2$ within this $\langle n\rangle=1.6$ ensemble. Nonetheless, these results confirm that both luminescence features seen in Figure 5B $(\langle n\rangle=1.6)$ derive from the same excited state.
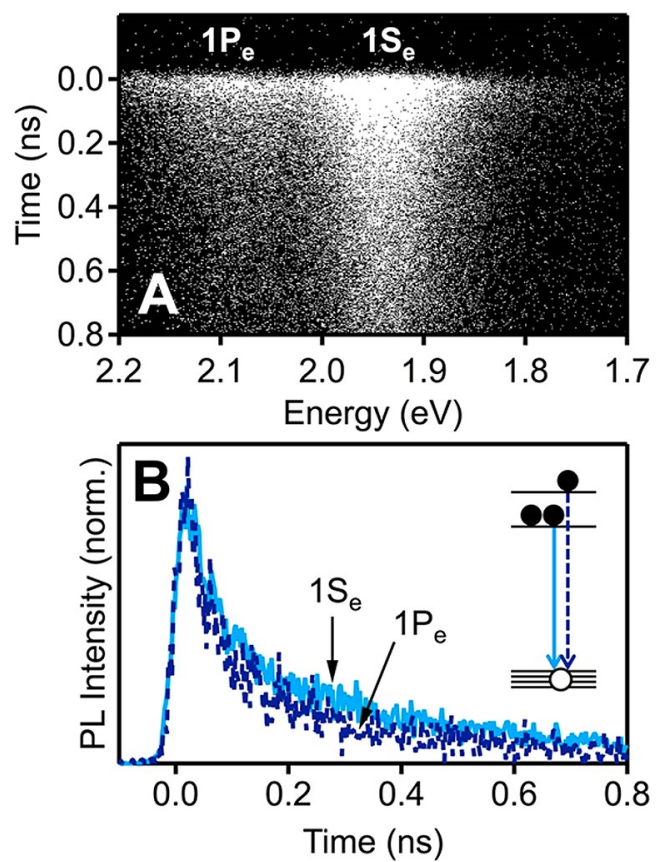

Figure 7. (A) Time-resolved PL streak image of maximally photodoped $(\langle\mathrm{n}\rangle=1.6) \mathrm{CdSe} / 2 \mathrm{CdS}$ QDs $\left(\lambda_{\mathrm{exc}}=405\right.$ $\mathrm{nm}, \mathrm{E}_{\mathrm{exc}}=3.06 \mathrm{eV}$ ). (B) Decay dynamics for $1 \mathrm{~S} \mathrm{e}$ (solid, integrated from 1.92 to $1.95 \mathrm{eV}$ ) and $1 \mathrm{Pe}$ (dashed, 2.07 to $2.12 \mathrm{eV}$ ) electrons in $\mathrm{CdSe} / 2 \mathrm{CdS}$, highlighting their very similar kinetics.

To explore the emission of these heavily n-doped QDs more systematically, we measured room-temperature PL decay dynamics of the CdSe/7CdS QDs at various values of $\langle n\rangle$. Figure 8 plots absorption spectra and PL decay curves of these CdSe/7CdS QDs poised at $\langle n\rangle=0,0.5$, and 1.8. The as-prepared QDs $(\langle n\rangle=0)$ exhibit purely excitonic emission, and their PL decay can be fit to a single exponential with a time constant $\tau_{\mathrm{X}}=35 \mathrm{~ns}$ (see Figure $\mathrm{S} 4$ for complete fit). When n-type carriers are introduced, PL is enhanced at short times due to the greater radiative rate of the negative trion. Faster decay of the PL is also observed, consistent with a combination of this increased radiative rate and the introduction of nonradiative trion Auger recombination. At $\langle n\rangle=0.5$, the PL decay is biexponential, reflecting a superposition of negative trion recombination $\left(\tau_{\mathrm{X}^{-}}=2.6 \mathrm{~ns}\right)$ and neutral exciton recombination $\left(\tau_{\mathrm{X}}\right)$ from the presence of both n-doped and undoped QDs in this ensemble. The value of $\tau_{\mathrm{X}^{-}}=2.6 \mathrm{~ns}$ is consistent with that predicted from the reported size dependence of $\tau_{X^{-}}$in photodoped n-type CdSe QDs ${ }^{15}$ using the effective QD diameter, as determined by the energy of the first excitonic absorption feature (see Figure S5). The PL decay is also biexponential at $\langle n\rangle=1.8$. Here, neutral exciton emission is completely absent because every QD possesses at least one excess $e_{\mathrm{CB}}^{-}$. Negative trion decay (with $\tau_{\mathrm{X}^{-}}$) is still observed, and in addition a faster process is 
observed with a decay constant of $\tau \approx 600 \mathrm{ps}$, or roughly $1 / 4 \tau_{\mathrm{X}^{-}}$. This faster component is interpreted as reflecting PL from QDs with $\langle n\rangle=2$ that have been photoexcited, thus containing four bandlike charge carriers (three $e_{\mathrm{CB}}^{-}$and one hole $h_{\mathrm{VB}}^{+}$) in their luminescent excited state, that is, a negative tetron $\left(\mathrm{X}^{2-}\right)$. The inset to Figure 8 illustrates carrier configurations relevant to each of the traces in panels $A$ and $B$. Trace 1 includes only neutral excitons $(\mathrm{X})$, trace 2 includes $\mathrm{X}$ and negative trions $\left(\mathrm{X}^{-}\right)$, and trace 3 includes $\mathrm{X}^{-}$and $\mathrm{X}^{2-}$. Upon reoxidation by air, both the absorption spectrum and PL dynamics return to their original values, being indistinguishable from those of the as-prepared QDs prior to photodoping. A small ( $\sim 19 \%$ relative) enhancement in overall PL quantum yield is observed after reoxidation, attributed to surface restructuring induced by this reduction/oxidation cycle.
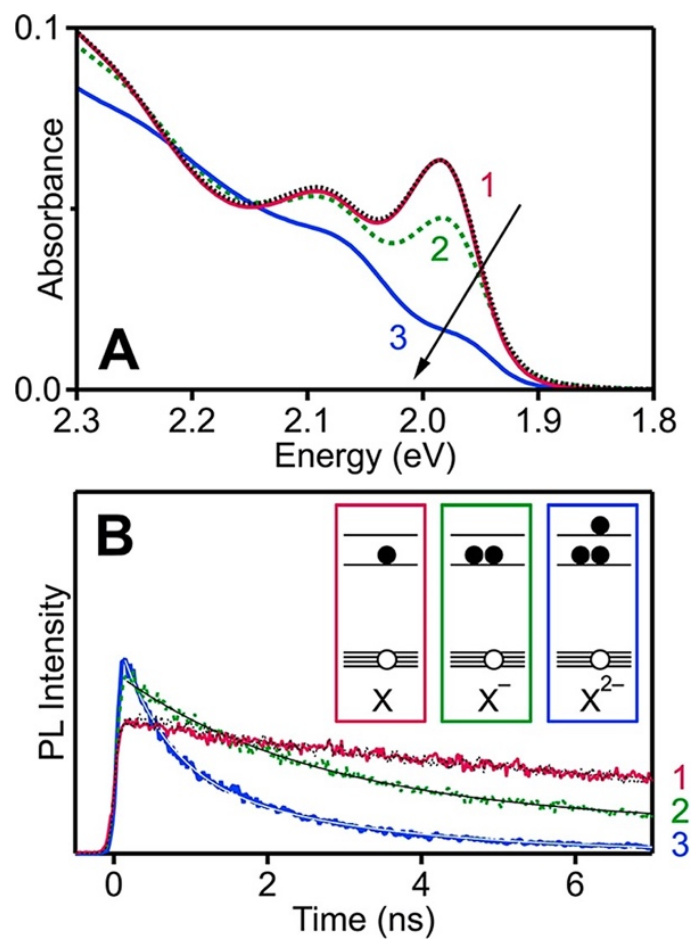

Figure 8. (A) Absorption spectra collected at three stages of photodoping for CdSe/7CdS QDs: (1) $\langle\mathrm{n}\rangle=0$, (2) $\langle\mathrm{n}\rangle=0.5$, and (3) $\langle\mathrm{n}\rangle=1.8$. The black dotted trace shows the spectrum collected after QD reoxidation to $\langle\mathrm{n}\rangle=0$ and is indistinguishable from the initial trace, demonstrating complete sample recovery. (B) PL decay curves corresponding to (1), (2), and (3) from panel A ( $\left.\lambda_{\mathrm{exc}}=405 \mathrm{~nm}, 3.06 \mathrm{eV}\right)$. The amplitude of the reoxidized curve (dotted) has been reduced (x 0.84 ) for clarity to account for mild sample brightening upon reoxidation. Thin solid traces illustrate biexponential fits for $\langle\mathrm{n}\rangle>0$ as described in the main text. Inset: Schematics illustrating the three relevant multicarrier configurations observed in panels A and B: neutral exciton, negative trion, and negative tetron (from left to right).

Figure 9 summarizes these findings. Figure 9A plots the fractional contributions from each of the carrier configurations to the PL dynamics vs $\langle n\rangle$ for the same CdSe/7CdS QDs, deduced from global fitting of a more extensive series of PL decay curves (see Figure S6 and Experimental Methods). As described above, at values of $\langle n\rangle=0$, the PL is entirely attributable 
to neutral $\mathrm{X}$. In the regime $0<\langle n\rangle<1$, the decay is biexponential with components corresponding to $\tau_{\mathrm{X}}$ and $\tau_{\mathrm{X}^{-}}$. At $\langle n\rangle>1$, a third component $\left(\tau^{2} \mathrm{X}^{-}\right)$is introduced.

Similarly, Figure 9B plots the fractional $1 \mathrm{~S}_{\mathrm{e}}$ and $1 \mathrm{P}_{\mathrm{e}}$ emission intensities vs $\langle n\rangle$ extracted from PL spectral fitting (see Figure S2). At $\langle n\rangle<1$, the $1 \mathrm{P}_{\mathrm{e}}$ emission is absent within experimental uncertainty, but when $\langle n\rangle>1$, the $1 \mathrm{P}_{\mathrm{e}}$ emission appears and increases in intensity. The relative intensity from the $1 \mathrm{~S}_{\mathrm{e}}$ emission is inversely correlated with that from the $1 \mathrm{P}_{\mathrm{e}}$ emission, decreasing only at $\langle n\rangle>1$. Overall, the PL QY decreases with increasing $\langle n\rangle$ (see Figure S6). The $1 \mathrm{~S}_{\mathrm{e}}$ data plotted in Figure $9 \mathrm{~B}$ compare well to the sum of $\mathrm{X}$ and $\mathrm{X}^{-}$decay amplitudes plotted in Figure 9A, whereas the $1 \mathrm{P}_{\mathrm{e}}$ data in Figure $9 \mathrm{~B}$ show the same trend as seen for the $\mathrm{X}^{2-}$ decay amplitudes in Figure 9A.

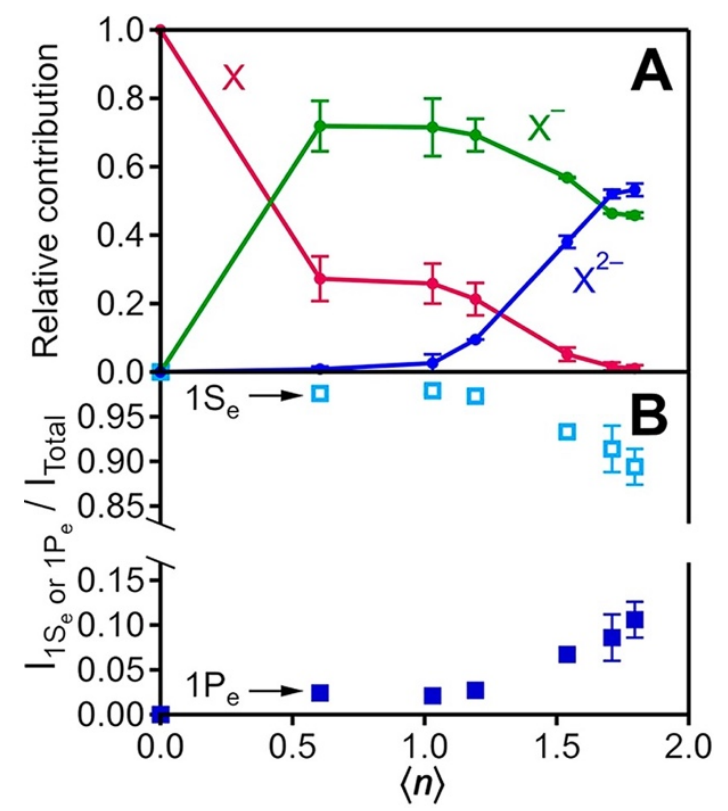

Figure 9. (A) Relative amplitudes of PL decay components arising from neutral excitons (X), negative trions $\left(\mathrm{X}^{-}\right)$, and negative tetrons $\left(\mathrm{X}^{2-}\right)$ in $\mathrm{n}$-doped CdSe/7CdS QDs plotted vs $\langle\mathrm{n}\rangle$, obtained from fitting of PL decay curves collected for each value of $\langle n\rangle$. (B) Intensities of PL peaks assigned to recombination of discrete $1 \mathrm{~S}_{\mathrm{e}}$ and $1 P_{e}$ electrons in $n$-doped CdSe/7CdS QDs plotted vs $\langle n\rangle$, determined by analysis of PL spectra collected for each value of $\langle\mathrm{n}\rangle$ (see Experimental Methods and Figure S2 for details).

The data in Figure 9 show that tetron luminescence, which can only appear when $\mathrm{n}=2$ in a given QD prior to photoexcitation, turns on rather abruptly at $\langle n\rangle>1$. Our previous studies have demonstrated diffusion-limited inter-QD electron transfer following such photodoping. ${ }^{17,30,31}$ Because of this rapid electron transfer, there is Fermi level equilibration within the ensemble of QDs such that the electron distributions among QDs are not determined by Poissonian photoexcitation statistics but rather by $\langle n\rangle$ and the ensemble's distributions in QD band-edge potentials and multielectron configurational energies. The abrupt appearance of tetron luminescence at $\langle n\rangle>1$ in Figure 9 thus reflects the tendency of the added electrons to 
distribute rather evenly across the ensemble of QDs, regardless of where they were initially generated.

From these PL decay dynamics, it is also possible to estimate radiative rate constants $\left(k_{r}\right)$, nonradiative Auger rate constants $\left(k_{A}\right)$, and PL QYs for the $\mathrm{X}, \mathrm{X}^{-}$, and $\mathrm{X}^{2-}$ electronic configurations of these QDs. Following prior analysis of multistate blinking in $\mathrm{CdSe} / \mathrm{CdS} \mathrm{QDs}$, these parameters can be quantified using eqs $1-3.8,25$ Here, $\beta$ is a carrier overlap factor that relates the radiative recombination rate of a charged exciton to that of the neutral exciton, and $i$ denotes the carrier configuration. Table 1 summarizes the values of these quantities determined from the dynamics measured for the CdSe/7CdS QDs (Figures 8 and S6).

Statistically, $k_{\mathrm{A}}^{\mathrm{X}^{2-}}$ should be $3 \mathrm{x}$ larger than $k_{\mathrm{A}}^{\mathrm{X}^{-}}$because of the increased number of possible recombination pairings, consistent with the factor of $\sim 4$ measured experimentally. Different radiative and nonradiative transition probabilities can be anticipated for recombination involving $1 \mathrm{~S}_{\mathrm{e}}$ and $1 \mathrm{P}_{\mathrm{e}}$ electrons, which will cause deviations from simple statistics. The values in Table 1 were obtained by using the integrated PL decay curves to estimate QYs at different values of $\langle n\rangle$ relative to the QY at $\langle n\rangle=0$, which was measured independently using an integrating sphere (see Figure S6).

$$
k_{\mathrm{r}}^{i}=\beta^{i} k^{\mathrm{X}}
$$

$$
\begin{aligned}
& k^{i}=k_{\mathrm{r}}^{i}+k_{\mathrm{A}}^{i}=1 / \tau_{i} \\
& \mathrm{QY}^{i}=\frac{k_{\mathrm{r}}^{i}}{k_{\mathrm{r}}^{i}+k_{\mathrm{A}}^{i}}
\end{aligned}
$$

Table 1. Recombination Parameters Determined for Various Neutral and Charged Excitonic Excited States of CdSe/7CdS QDs

\begin{tabular}{llllll}
$\boldsymbol{i}$ & $\mathbf{Q Y}$ & $\boldsymbol{\tau}_{\boldsymbol{i}}(\mathbf{n s})$ & $\mathbf{1} / \boldsymbol{k}_{\mathbf{r}}^{\boldsymbol{i}}(\mathbf{n s})$ & $\mathbf{1} / \boldsymbol{k}_{\mathbf{A}}^{\boldsymbol{i}}(\mathbf{n s})$ & $\boldsymbol{\beta}^{\boldsymbol{i}}$ \\
\hline $\mathrm{X}$ & $0.73^{\mathrm{a}}$ & 35 & 35 & - & 1 \\
$\mathrm{X}^{-}$ & 0.11 & 2.6 & 23.6 & 2.9 & 1.48 \\
$\mathrm{X}^{2-}$ & 0.06 & 0.6 & 10 & 0.6 & 3.5 \\
\hline
\end{tabular}

a Measured independently using an integrating sphere.

The $\beta$ values determined here yield information about the Coulomb forces acting between carriers. The experimental $\beta^{\mathrm{X}^{-}}$value is smaller than the statistical expectation $\left(\beta^{\mathrm{X}^{-}}=2\right)$, and it is also smaller than the value observed in CdSe QDs $\left(\beta^{\mathrm{X}^{-}}=2.2\right),{ }^{32}$ but it agrees well with the value measured for CdSe/CdS QDs overcoated with silica $\left(\beta^{\mathrm{X}^{-}-}=1.49\right)$. This reduced value of 
$\beta^{\mathrm{X}^{-}}$is consistent with diminished carrier overlap in these core/shell QDs because of proper core/shell band alignment for electron but not hole delocalization. ${ }^{25}$ The value of $\beta^{\mathrm{X}^{2-}}$ in Table 1 is comparable to but slightly larger than that expected purely statistically $\left(\beta^{\mathrm{X}^{2-}}=3\right)$.

\section{Discussion}

The results and analysis presented above show that growth of CdS shells around colloidal CdSe QDs markedly improves the stability of excess conduction-band electrons added via photodoping. The resulting colloidal n-type QDs can be stabilized for hours in solution at room temperature and are thus amenable to investigation by various chemical and spectroscopic methods. Similar to unshelled CdSe QDs, ${ }^{21}$ we find that the maximum number of conductionband electrons that can be added to these core/shell QDs via photodoping, $\left\langle n_{\max }\right\rangle$, increases with increasing QD diameter across a well-controlled series of related QDs. For the present QDs, we can add as many as two excess electrons per QD via photodoping, but not more. This maximum is confirmed by direct chemical titration of the excess electrons. This value of $\left\langle n_{\max }\right\rangle$ is somewhat larger than we found for unshelled CdSe QDs $\left(\left\langle\left\langle n_{\max }\right\rangle<\sim 1.6\right),{ }^{18,21}\right.$ but not dramatically so. The greatest contrast between shelled and unshelled CdSe QDs is in the longterm stability of these excess electrons. An interesting question pertains to the precise origin of $\left\langle n_{\max }\right\rangle$, which remains unclear. We have previously demonstrated ${ }^{18,21}$ that $\left\langle n_{\max }\right\rangle$ for (unshelled) CdSe QDs is not related to the specific orbital occupancies of the QDs in any way, because in some cases $\left\langle n_{\max }\right\rangle$ occurs at values well below 1, meaning many QDs within the ensemble actually possess no conduction-band electrons at maximum photodoping. Such data suggest an important role of surface traps in determining the most-negative Fermi level achievable during photodoping. ${ }^{18,21}$ For the present CdSe/CdS QDs, $\left\langle n_{\max }\right\rangle$ reaches nearly 2 with the thickest of our CdS shells $(x=7)$, but we see no evidence for steady-state occupancy of the $1 \mathrm{P}_{\mathrm{e}}$ levels in either the PL spectra or the PL decay data. We hypothesize that such $1 \mathrm{P}_{\mathrm{e}}$ electrons, which would be at least $\sim 150-300 \mathrm{meV}$ (variable with quantum confinement, Figure 2) less stable than the $1 \mathrm{~S}_{\mathrm{e}}$ electrons, trap at much faster rates than the $1 \mathrm{~S}_{\mathrm{e}}$ electrons even in these core/shell heterostructures because of a greater trap density of states at these potentials, and this rapid trapping precludes stable $1 \mathrm{P}_{\mathrm{e}}$ occupancy. Maximum carrier densities are thus limited to $\langle N\rangle \sim 10^{19} \mathrm{~cm}^{-3}$ in these $\mathrm{CdSe} / \mathrm{CdS}$ QDs, compared to an order of magnitude larger carrier densities found for photodoped metal-oxide $\left(\mathrm{ZnO}, \mathrm{In}_{2} \mathrm{O}_{3}\right.$, and related) nanocrystals. ${ }^{13,33}$

Previously, remote chemical dopants were also found to introduce no more than two excess electrons per QD into CdSe QDs. ${ }^{14}$ Interestingly, even when electrons are introduced 
electrochemically, CdSe QD films still allow only partial filling of the $1 \mathrm{P}_{\mathrm{e}}$ shell. ${ }^{34}$ It is likely that both of these observations also reflect the accessibility of surface electron traps at the fairly negative potentials of the $1 \mathrm{P}_{\mathrm{e}}$ electrons. During preparation of this manuscript, another study of the same n-type photodoping of $\mathrm{CdSe} / \mathrm{CdS}$ QDs using $\mathrm{Li}\left[\mathrm{Et}_{3} \mathrm{BH}\right]$ was published in which values as large as $\langle n\rangle=6$ are reported. ${ }^{22}$ It is conceivable that in these QDs, the density of surface traps was diminished significantly compared to the other samples discussed above, allowing accumulation of many more electrons per QD before reaching the potentials of these traps. We note, however, that $\langle n\rangle$ values in ref 22 were determined by analysis of the first exciton's absorption bleach under the assumption that distributions of excess electrons among QDs reflect the Poissonian QD photoexcitation statistics, without consideration of subsequent inter-QD electron transfer. Under this assumption, QDs with very large values of $n$ coexist with QDs having no excess electrons, and much larger values of $\langle n\rangle$ are thus required to fully bleach the first excitonic absorption feature. Only the $1 \mathrm{~S}_{\mathrm{e}}$ electrons contribute to this bleach. Such a broad Poissonian distribution of electrons among QDs appears inconsistent with our previous observations of rapid inter-QD electron transfer, ${ }^{17,30,31}$ the abrupt appearance of tetron PL when increasing from $\langle n\rangle<1$ to $\langle n\rangle>1$ (Figure 9), and the confirmation by direct chemical titration that a full bleach of the first excitonic absorption feature is achieved at $\langle n\rangle \sim 2$ (Figure 4).

More generally, the capability to incorporate multiple, robust n-type carriers into highoptical-quality colloidal CdSe QDs via photochemical reduction described here presents exciting opportunities to investigate the various physical properties of these unusual materials. For example, long-lived spin coherence has been demonstrated in a host of singly reduced semiconductor QDs generated as films on electrode surfaces. ${ }^{35}$ In the case of the tetrons described here, the spin configuration reduces to that of a neutral exciton because the excess carriers form a paired singlet with zero spin. These negative tetrons may therefore prove of fundamental interest from the standpoint that the one unpaired electron occupies the $1 \mathrm{P}_{\mathrm{e}}$ orbital rather than the $1 \mathrm{Se}$ orbital, and spin-sensitive techniques such as time-resolved Faraday rotation (TRFR) or magnetoluminescence could thus be used to selectively probe specifically the $1 \mathrm{P}_{\mathrm{e}}$ electron. TRFR experiments on electrochemically reduced CdSe QDs do demonstrate a convergence of two $g$ values in neutral QDs to a single resonance under negative bias, ${ }^{36}$ attributed to suppression of excitonic precession and demonstrating feasibility of such measurements on $n$-doped QDs. Stable photodoping may also help advance the understanding of dopant-carrier magnetic-exchange interactions involving impurity spin centers in colloidal CdSe QDs. For example, photodoping of $\mathrm{Zn}_{1_{-x}} \mathrm{Mn}_{\mathrm{x}} \mathrm{O}$ QDs was shown to induce long-range 
carrier-mediated $\mathrm{Mn}^{2+}-\mathrm{Mn}^{2+}$ exchange coupling ${ }^{37}$ and modify $\mathrm{Mn}^{2+}$ spin-relaxation rates. ${ }^{38}$ Similar experiments in $\mathrm{Cd}_{1-\mathrm{x}} \mathrm{Mn}_{\mathrm{x}} \mathrm{Se}$ QDs could take advantage of the ability to retain excitonic PL in these doped QDs, ${ }^{39,40}$ allowing use of PL and magneto-PL to probe such exchange interactions. All of these experiments will benefit from the ability to generate and stabilize excess charge carriers in high-optical-quality n-doped CdSe-based QDs.

\section{Conclusion}

Colloidal CdSe/CdS core/shell QDs were photochemically reduced, incorporating up to two conduction-band electrons per QD. The maximum level of n-doping was shown to increase as a function of shell thickness, reflecting suppression of surface electron trapping. CdS shelling vastly enhances carrier stability in colloidal CdSe QDs, with excess electrons stable on time scales of hours in solution at room temperature, which in turn enables accurate quantification of the number of excess charge carriers per QD via direct chemical titration. Discrete emission bands involving recombination of $1 \mathrm{~S}_{\mathrm{e}}$ and $1 \mathrm{P}_{\mathrm{e}}$ electrons are observed at values of $\langle n\rangle>1$, both decaying with similar rate constants, indicating formation of four-carrier negative tetrons. Analysis of the PL decay dynamics confirms this assignment. These results highlight the exceptional control afforded by photodoping for incorporating excess conductionband electrons into freestanding colloidal CdSe/CdS QDs and suggest opportunities for development of novel nanomaterials with tailored electronic configurations relevant to future applications in optoelectronics, photoredox chemistry, and other technologies.

\section{Experimental Methods}

General Considerations. Unless stated otherwise, all syntheses and sample preparations were performed using standard Schlenk line technique under a dinitrogen atmosphere, or in a nitrogen-filled glovebox with anhydrous solvents.

Chemicals. Cadmium oxide (CdO; 99.99+\%), 1-octadecene (ODE; 90\%), oleic acid (OA; 90\%), hexadecylamine (HDA; 90\%), trioctylphosphine oxide (TOPO; 99\%), selenium metal (Se; 99.99+\%), and 1-octanethiol (OT; $>98.5 \%$ ) were purchased from Sigma-Aldrich. Trioctylphosphine (TOP; 97\%) was purchased from Strem. All chemicals were used as received.

Quantum Dot Synthesis. Colloidal CdSe QDs were synthesized using a hot-injection procedure adapted from ref 41 . In a 100-mL 3-neck round bottom flask, a stirred solution of 
$150 \mathrm{mg}$ of $\mathrm{CdO}, 12 \mathrm{~g}$ of $\mathrm{ODE}$, and $1 \mathrm{~g}$ of $\mathrm{OA}$ was degassed under vacuum at $110^{\circ} \mathrm{C}$ for 1 hour. The solution was then heated to $\sim 280^{\circ} \mathrm{C}$ under $\mathrm{N}_{2}$ until the red solution turned clear and colorless. The solution was allowed to cool to $100^{\circ} \mathrm{C}$ and was then placed under vacuum for 1 hour to remove $\mathrm{O}_{2}$ produced by forming the $\mathrm{Cd}(\text { oleate })_{2}$. $2 \mathrm{~g}$ of HDA and $2 \mathrm{~g}$ of TOPO were then added to the flask under an overpressure of $\mathrm{N}_{2}$, followed by 30 minutes of vacuum and 3 purge cycles with $\mathrm{N}_{2}$. After purging the system, the flask was heated to $300^{\circ} \mathrm{C}$ under $\mathrm{N}_{2}$. In a separate $25-\mathrm{mL}$ flask, $190 \mathrm{mg}$ of Se was dissolved in $4 \mathrm{~mL}$ of TOP and then rapidly injected into the hot $\mathrm{Cd}$ (oleate)2 solution. After 5 minutes, the heating mantle was removed and the cooled solution of QDs was purified by flocculation with ethanol and methanol followed by resuspension in toluene.

$\mathrm{CdS}$ shells were grown onto the CdSe cores using a procedure adapted from ref 7 . For a typical shell growth, a $0.16 \mathrm{M} \mathrm{Cd}($ oleate) 2 solution in ODE and a $0.20 \mathrm{M}$ solution of OT in ODE were separately injected at $2.5 \mathrm{~mL} /$ hour to a degassed solution containing $200 \mathrm{nmol}$ of CdSe cores in $4 \mathrm{~g}$ of $\mathrm{ODE}$ and $2 \mathrm{~g}$ of $\mathrm{HDA}$ at $200^{\circ} \mathrm{C}$. Once the injection was started, the temperature of the solution was raised to $300^{\circ} \mathrm{C}$ and held there until the injection was complete. The total volume of each solution injected was varied depending on the desired shell thickness, while the volumes for the two shell precursor solutions were kept equal. After the injection was complete, the solution was cooled to room temperature and the QDs purified by flocculation with ethanol and methanol followed by resuspension in toluene.

Physical Characterization. Transmission electron miscroscope (TEM) images were obtained using a FEI Tecnai G2 F20 operating at $200 \mathrm{kV}$. Quantum yields were measured on colloidal QD suspensions by exciting samples with a Xe lamp coupled to a monochromator $\left(\lambda_{\text {exc }}=450\right.$ $\mathrm{nm}$ ) and detecting in an external quantum efficiency integrating sphere (Hamamatsu C992012). All spectroscopic measurements were performed at room temperature on suspensions of colloidal QDs in toluene. Absorption spectra were measured in a Cary 500 (Varian) spectrophotometer. QD concentrations were determined using the absorption intensity at the first excitonic peak and a calibration curve for CdSe QDs. ${ }^{42}$ For infrared (IR) absorption measurements, QDs were mixed with excess $\mathrm{LiBEt}_{3} \mathrm{H}$ in the dark in a nitrogen-filled glovebox, and the solution loaded into an air-free IR cell with either $\mathrm{CaF}_{2}(\mathrm{CdSe} / 0 \mathrm{CdS}$ and $\mathrm{CdSe} / 2 \mathrm{CdS}$ in toluene) or $\mathrm{KBr}(\mathrm{CdSe} / 7 \mathrm{CdS}$ in cyclohexane) windows separated by a $100 \mu \mathrm{m}$ Teflon spacer. Measurements were collected on an FTIR spectrometer (ThermoScientific Nicolet-8700) with a $\mathrm{HgCdTe}$ detector. Continuous-wave PL spectra were measured by exciting the sample with a $5 \mathrm{~mW} 405 \mathrm{~nm}$ laser pointer and detecting on an OceanOptics 2000+ spectrometer. Time- 
resolved PL measurements were performed using the frequency-doubled output of a Ti:sapphire laser at a $200-\mathrm{kHz}$ repetition rate (405 nm, $150 \mathrm{fs}$ pulse, $1.2-1.5 \mathrm{~nJ} / \mathrm{pulse})$. Decay curves were measured using a monochromator and streak camera with an instrument response function of $\sim 15$ ps.

Photodoping. Photodoping was performed following the method introduced in ref 14. QDs were dried and resuspended in anhydrous toluene in a nitrogen-filled glovebox prior to photodoping experiments. Samples were diluted to the desired concentration (generally $\sim 100$ $\mathrm{nM}$ for PL experiments), and 50-1000 equivalents of $\mathrm{Li}\left[\mathrm{BEt}_{3} \mathrm{H}\right]$ were added. Samples were irradiated with a $5 \mathrm{~mW} 405 \mathrm{~nm}$ laser pointer to achieve desired level of photodoping. To achieve maximal photodoping, $\mathrm{LiBEt}_{3} \mathrm{H}$ was often added in small increments to mitigate sample scattering and etching.

Determination of $\langle\boldsymbol{n}\rangle$. A solution of $\mathrm{CdSe} / 7 \mathrm{CdS}$ QDs in toluene was prepared in a nitrogenfilled glovebox and measured in a $1 \mathrm{~cm}$ pathlength quartz cuvette. Spectra were collected using an OceanOptics $2000+$ spectrophotometer with an absorption attachment. $\sim 200$ equiv of $\mathrm{LiBEt}_{3} \mathrm{H}$ was added, and photodoping was performed using the OceanOptics light source for photoexcitation until no additional bleach was observed over the course of $30 \mathrm{~s}$. These excess electrons were then quantified via titration by addition of $10-\mu \mathrm{L}$ aliquots of $9 \mu \mathrm{M}$ $\left[\mathrm{FeCp}_{2}{ }_{2}\right]\left[\mathrm{BArF}_{4}\right]$ in THF. The light source was switched off during aliquot additions to avoid in situ photodoping between spectral collections. The titration took $<10 \mathrm{~min}$ to complete, and thus anaerobic recovery for this thick-shelled sample was considered negligible over the course of the experiment.

Anaerobic Recovery. A series of QD samples was photodoped with careful attention paid to minimize differences in sample handling. The samples were prepared with the same optical density at their respective first absorption peak maxima in a solution of $30 \mu \mathrm{M} \mathrm{Li}\left[\mathrm{Et}_{3} \mathrm{BH}\right]$ in toluene. Each was prepared anaerobically in a quartz cuvette equipped with a magnetic stir bar and sealed with a Teflon stopper. A photolysis lamp paired with a monochromator was tuned to selectively photoexcite each sample at its respective first excitonic absorption feature, and the light was focused onto the cuvette, which was set to stir inside the Cary spectrophotometer sample cavity. The power of the photolysis light was the same for all samples $(0.5 \mathrm{~mW})$, and each sample was irradiated for a total of $30 \mathrm{~min}$. After $30 \mathrm{~min}$, the lamp was switched off, and absorption scans were intermittently collected to monitor the rate of anaerobic recovery. 
Spectral Analysis. Absorption spectra at different values of $\langle n\rangle$ were fit to a sum of four Gaussians using a global fitting procedure. Widths were fixed, but energies and intensities were allowed to float to reflect the extent of photodoping and electron accumulation. For analysis of the PL spectra, the spectrum of neutral $(\langle n\rangle=0) \mathrm{CdSe} / 7 \mathrm{CdS}$ QDs was fitted. Upon photodoping and with increasing $\langle n\rangle$, this band was allowed to shift to lower energy as a new fixed-width Gaussian feature (ascribed to emission involving recombination of $1 \mathrm{P}_{\mathrm{e}}$ electrons) gained intensity at $2.01 \mathrm{eV}$. For PL decay analysis, global triexponential fitting of the PL decay curves collected for the $\mathrm{CdSe} / 7 \mathrm{CdS}$ QD sample at various photodoping levels yielded the fitted values of $\tau$ corresponding to $\mathrm{X}, \mathrm{X}^{-}$, and $\mathrm{X}^{2-}$ listed in Table 1 , and the fitted $\mathrm{X}, \mathrm{X}^{-}$, and $\mathrm{X}^{2-}$ amplitudes plotted vs $\langle n\rangle$ in Figure 9A.

\section{Author Information}

Corresponding Author:*E-mail: gamelin@chem.washington.edu.

Present Addresses: §Department of Chemistry and Biochemistry, 251 Nieuwland Science Hall, Notre Dame, IN 46556, United States.

$\$$ Laboratory for Fundamental BioPhotonics (LBP), Institute of Bioengineering (IBI) and Institute of Materials Science (IMX), School of Engineering (STI), École Polytechnique Fédérale de Lausanne (EPFL), CH-1015 Lausanne, Switzerland.

\section{Acknowledgments}

This research was supported by the NSF (CHE-1506014 to D.R.G., Graduate Research Fellowship DGE-1256082 to K.H.H.), the State of Washington through the University of Washington Clean Energy Institute Fellowship Program (to C.S.E.), the NIH (Postdoctoral Fellowship F32GM110876 to E.Y.T.), and the Swiss National Science Foundation (Early Postdoctoral Mobility Fellowship to A.M.). The authors thank Michael C. De Siena for TEM images and valuable technical support. Part of this work was conducted at the Molecular Analysis Facility, a National Nanotechnology Coordinated Infrastructure site at the University of Washington, which is supported in part by the National Science Foundation (grant ECC1542101), the University of Washington, the Molecular Engineering and Sciences Institute, the Clean Energy Institute, and the National Institutes of Health.

\section{References}


(1) Peng, X.; Schlamp, M. C.; Kadavanich, A. V.; Alivisatos, A. P. Epitaxial Growth of Highly Luminescent $\mathrm{CdSe} / \mathrm{CdS}$ Core/Shell Nanocrystals with Photostability and Electronic Accessibility. J. Am. Chem. Soc. 1997, 119, 7019-7029.

(2) Li, J. J.; Wang, Y. A.; Guo, W.; Keay, J. C.; Mishima, T. D.; Johnson, M. B.; Peng, X. Large-Scale Synthesis of Nearly Monodisperse CdSe/CdS Core/Shell Nanocrystals Using AirStable Reagents via Successive Ion Layer Adsorption and Reaction. J. Am. Chem. Soc. 2003, $125,12567-12575$.

(3) Mahler, B.; Spinicelli, P.; Buil, S.; Quelin, X.; Hermier, J.-P.; Dubertret, B. Towards NonBlinking Colloidal Quantum Dots. Nat. Mater. 2008, 7, 659-664.

(4) Chen, Y.; Vela, J.; Htoon, H.; Casson, J. L.; Werder, D. J.; Bussian, D. A.; Klimov, V. I.; Hollingsworth, J. A. "Giant" Multishell CdSe Nanocrystal Quantum Dots with Suppressed Blinking. J. Am. Chem. Soc. 2008, 130, 5026-5027.

(5) Malko, A. V.; Park, Y.-S.; Sampat, S.; Galland, C.; Vela, J.; Chen, Y.; Hollingsworth, J. A.; Klimov, V. I.; Htoon, H. Pump-Intensity- and Shell-Thickness-Dependent Evolution of Photoluminescence Blinking in Individual Core/Shell CdSe/CdS Nanocrystals. Nano Lett. 2011, 11, 5213-5218.

(6) Galland, C.; Ghosh, Y.; Steinbrück, A.; Hollingsworth, J. A.; Htoon, H.; Klimov, V. I. Lifetime Blinking in Nonblinking Nanocrystal Quantum Dots. Nat. Commun. 2012, 3, 908.

(7) Chen, O.; Zhao, J.; Chauhan, V. P.; Cui, J.; Wong, C.; Harris, D. K.; Wei, H.; Han, H.-S.; Fukumura, D.; Jain, R. K.; Bawendi, M. G. Compact High-Quality CdSe-CdS Core-Shell Nanocrystals with Narrow Emission Linewidths and Suppressed Blinking. Nat. Mater. 2013, $12,445-451$.

(8) Park, Y.-S.; Bae, W. K.; Pietryga, J. M.; Klimov, V. I. Auger Recombination of Biexcitons and Negative and Positive Trions in Individual Quantum Dots. ACS Nano 2014, 8, 7288-7296.

(9) Rabouw, F. T.; Vaxenburg, R.; Bakulin, A. A.; van Dijk-Moes, R. J. A.; Bakker, H. J.; Rodina, A.; Lifshitz, E.; Efros, A. L.; Koenderink, A. F.; Vanmaekelbergh, D. Dynamics of Intraband and Interband Auger Processes in Colloidal Core-Shell Quantum Dots. ACS Nano 2015, 9, 10366-10376.

(10) Ehrat, F.; Simon, T.; Stolarczyk, J. K.; Feldmann, J. Size Effects on Photocatalytic H2 Generation with CdSe/CdS Core-Shell Nanocrystals. J. Phys. Chem. 2015, 229, 205-219.

(11) Elimelech, O.; Liu, J.; Plonka, A. M.; Frenkel, A. I.; Banin, U. Size Dependence of Doping via Vacancy Formation in Copper Sulfide Nanocrystals. Angew. Chem., Int. Ed. 2017, 56, $10335-10340$.

(12) Schimpf, A. M.; Knowles, K. E.; Carroll, G. M.; Gamelin, D. R. Electronic Doping and Redox Potential Tuning in Colloidal Semiconductor Nanocrystals. Acc. Chem. Res. 2015, 48, 1929-1937. 
(13) Schimpf, A. M.; Gunthardt, C. E.; Rinehart, J. D.; Mayer, J. M.; Gamelin, D. R. Controlling Carrier Densities in Photochemically Reduced Colloidal ZnO Nanocrystals: Size Dependence and Role of the Hole Quencher. J. Am. Chem. Soc. 2013, 135, 16569-16577.

(14) Rinehart, J. D.; Schimpf, A. M.; Weaver, A. L.; Cohn, A. W.; Gamelin, D. R. Photochemical Electronic Doping of Colloidal CdSe Nanocrystals. J. Am. Chem. Soc. 2013, 135, 18782-18785.

(15) Cohn, A. W.; Rinehart, J. D.; Schimpf, A. M.; Weaver, A. L.; Gamelin, D. R. Size Dependence of Negative Trion Auger Recombination in Photodoped CdSe Nanocrystals. Nano Lett. 2014, 14, 353-358.

(16) Schimpf, A. M.; Thakkar, N.; Gunthardt, C. E.; Masiello, D. J.; Gamelin, D. R. ChargeTunable Quantum Plasmons in Colloidal Semiconductor Nanocrystals. ACS Nano 2014, 8, 1065-1072.

(17) Carroll, G. M.; Brozek, C. K.; Hartstein, K. H.; Tsui, E. Y.; Gamelin, D. R. Potentiometric Measurements of Semiconductor Nanocrystal Redox Potentials. J. Am. Chem. Soc. 2016, 138, 4310-4313.

(18) Carroll, G. M.; Tsui, E. Y.; Brozek, C. K.; Gamelin, D. R. Spectroelectrochemical Measurement of Surface Electrostatic Contributions to Colloidal CdSe Nanocrystal Redox Potentials. Chem. Mater. 2016, 28, 7912-7918.

(19) Tsui, E. Y.; Hartstein, K. H.; Gamelin, D. R. Selenium Redox Reactivity on Colloidal CdSe Quantum Dot Surfaces. J. Am. Chem. Soc. 2016, 138, 11105-11108.

(20) Brozek, C. K.; Hartstein, K. H.; Gamelin, D. R. Potentiometric Titrations for Measuring the Capacitance of Colloidal Photodoped ZnO Nanocrystals. J. Am. Chem. Soc. 2016, 138, 10605-10610.

(21) Tsui, E. Y.; Carroll, G. M.; Miller, B.; Marchioro, A.; Gamelin, D. R. Extremely Slow Spontaneous Electron Trapping in Photodoped n-Type CdSe Nanocrystals. Chem. Mater. 2017, 29, 3754-3762.

(22) Wu, K.; Lim, J.; Klimov, V. I. Superposition Principle in Auger Recombination of Charged and Neutral Multicarrier States in Semiconductor Quantum Dots. ACS Nano 2017, $11,8437-8447$.

(23) Wang, C.; Wehrenberg, B. L.; Woo, C. Y.; Guyot-Sionnest, P. Light Emission and Amplification in Charged CdSe Quantum Dots. J. Phys. Chem. B 2004, 108, 9027-9031.

(24) Jha, P. P.; Guyot-Sionnest, P. Photoluminescence Switching of Charged Quantum Dot Films. J. Phys. Chem. C 2007, 111, 15440-15445.

(25) Sampat, S.; Karan, N. S.; Guo, T.; Htoon, H.; Hollingsworth, J. A.; Malko, A. V. Multistate Blinking and Scaling of Recombination Rates in Individual Silica-Coated CdSe/CdS Nanocrystals. ACS Photonics 2015, 2, 1505-1512. 
(26) Coropceanu, I.; Bawendi, M. G. Core/Shell Quantum Dot Based Luminescent Solar Concentrators with Reduced Reabsorption and Enhanced Efficiency. Nano Lett. 2014, 14, 4097-4101.

(27) Steiner, D.; Dorfs, D.; Banin, U.; Della Sala, F.; Manna, L.; Millo, O. Determination of Band Offsets in Heterostructured Colloidal Nanorods Using Scanning Tunneling Spectroscopy. Nano Lett. 2008, 8, 2954-2958.

(28) Carroll, G. M.; Tsui, E. Y.; Brozek, C. K.; Gamelin, D. R. Spectroelectrochemical Measurement of Surface Electrostatic Contributions to Colloidal CdSe Nanocrystal Redox Potentials. Chem. Mater. 2016, 28, 7912-7918.

(29) Saba, M.; Aresti, M.; Quochi, F.; Marceddu, M.; Loi, M. A.; Huang, J.; Talapin, D. V.; Mura, A.; Bongiovanni, G. Light-Induced Charged and Trap States in Colloidal Nanocrystals Detected by Variable Pulse Rate Photoluminescence Spectroscopy. ACS Nano 2013, 7, 229-238.

(30) Hayoun, R.; Whitaker, K. M.; Gamelin, D. R.; Mayer, J. M. Electron Transfer Between Colloidal ZnO Nanocrystals. J. Am. Chem. Soc. 2011, 133, 4228-4231.

(31) Cohn, A. W.; Janßen, N.; Mayer, J. M.; Gamelin, D. R. Photocharging ZnO Nanocrystals: Picosecond Hole Capture, Electron Accumulation, and Auger Recombination. J. Phys. Chem. C 2012, 116, 20633-20642.

(32) Jha, P. P.; Guyot-Sionnest, P. Trion Decay in Colloidal Quantum Dots. ACS Nano 2009, $3,1011-1015$.

(33) Schimpf, A. M.; Runnerstrom, E. L.; Lounis, S. D.; Milliron, D. J.; Gamelin, D. R. Redox Energies and Plasmon Resonance Energies of Photodoped $\mathrm{In}_{2} \mathrm{O}_{3}$ and Sn-doped $\operatorname{In}_{2} \mathrm{O}_{3}$ Nanocrystals. J. Am. Chem. Soc. 2015, 137, 518-524.

(34) Yu, D.; Wang, C.; Guyot-Sionnest, P. n-Type Conducting CdSe Nanocrystal Solids. Science 2003, 300, 1277-1280.

(35) Kalevich, V. K.; Merkulov, I. A.; Shiryaev, A. Y.; Kavokin, K. V.; Ikezawa, M.; Okuno, T.; Brunkov, P. N.; Zhukov, A. E.; Ustinov, V. M.; Masumoto, Y. Optical Spin Polarization and Exchange Interaction in Doubly Charged InAs Self-Assembled Quantum Dots. Phys. Rev. B: Condens. Matter Mater. Phys. 2005, 72, 045325.

(36) Stern, N. P.; Poggio, M.; Bartl, M. H.; Hu, E. L.; Stucky, G. D.; Awschalom, D. D. Spin Dynamics in Electrochemically Charged CdSe Quantum Dots. Phys. Rev. B: Condens. Matter Mater. Phys. 2005, 72, 161303.

(37) Ochsenbein, S. T.; Feng, Y.; Whitaker, K. M.; Badaeva, E.; Liu, W. K.; Li, X.; Gamelin, D. R. Charge-Controlled Magnetism in Colloidal Doped Semiconductor Nanocrystals. Nat. Nanotechnol. 2009, 4, 681-687.

(38) Schimpf, A. M.; Rinehart, J. D.; Ochsenbein, S. T.; Gamelin, D. R. Charge-State Control of $\mathrm{Mn}^{2+}$ Spin Relaxation Dynamics in Colloidal n-Type $\mathrm{Zn}_{1-\mathrm{x}} \mathrm{Mn}_{\mathrm{x}} \mathrm{O}$ Nanocrystals. J. Phys. Chem. Lett. 2015, 6, 1748-1753. 
(39) Beaulac, R.; Archer, P. I.; Ochsenbein, S. T.; Gamelin, D. R. $\mathrm{Mn}^{2+}$-Doped CdSe Quantum Dots: New Inorganic Materials for Spin-Electronics and Spin-Photonics. Adv. Funct. Mater. 2008, 18, 3873-3891.

(40) Nelson, H. D.; Bradshaw, L. R.; Barrows, C. J.; Vlaskin, V. A.; Gamelin, D. R. Picosecond Dynamics of Excitonic Magnetic Polarons in Colloidal Diffusion-Doped $\mathrm{Cd}_{1-\mathrm{x}} \mathrm{Mn}_{\mathrm{x}} \mathrm{Se}$ Quantum Dots. ACS Nano 2015, 9, 11177-11191.

(41) Qu, L.; Peng, X. Control of Photoluminescence Properties of CdSe Nanocrystals in Growth. J. Am. Chem. Soc. 2002, 124, 2049-2055.

(42) Yu, W. W.; Qu, L.; Guo, W.; Peng, X. Experimental Determination of the Extinction Coefficient of CdTe, CdSe, and CdS Nanocrystals. Chem. Mater. 2003, 15, 2854-2860. 